\title{
Sarcoidal Granulomas in Cytological Specimens from Intrathoracic Adenopathy: Morphologic Characteristics and Radiographic Correlations
}

\author{
Alessandra Cancellieri ${ }^{b} \quad$ Kevin O. Leslie $^{a} \quad$ Carmine Tinelli $^{d}$ Marco Patelli ${ }^{c}$ \\ Rocco Trisolinic \\ ${ }^{a}$ Department of Laboratory Medicine and Pathology, Mayo Clinic Arizona, Scottsdale, Ariz., USA; \\ ${ }^{b}$ Anatomic Pathology Unit and ${ }^{C}$ Thoracic Endoscopy and Pulmonology Unit, Maggiore Hospital, Bologna, and \\ ${ }^{\mathrm{d} C l i n i c a l}$ Epidemiology and Biometry Service, IRCCS Policlinico San Matteo, Pavia, Italy
}

\section{Key Words}

Cytology · Granuloma • Lymphadenopathy • Sarcoidosis •

Transbronchial needle aspiration · Endobronchial

ultrasound-guided transbronchial needle aspiration •

Tuberculosis

\begin{abstract}
Background: Clinical experience and literature data suggest that the ability of pathologists to identify granulomas in cytological specimens from intrathoracic lymphadenopathy varies considerably and may negatively influence the yield of transbronchial needle aspiration (TBNA), both conventional and ultrasound-guided (EBUS-TBNA). Objectives: To describe the cytomorphology of sarcoidal granulomas on TBNA cytology specimens and to analyze the presence of associations between the cytological characteristics of granulomas and the radiographic stage of sarcoidosis. Methods: TBNA cytological specimens from 123 sarcoidosis patients and 14 tuberculosis patients (control population) were reviewed independently by two pathologists blinded to the clinical-radiological details. Results: Sarcoidal granulomas were small [median (IQR) largest diameter: 0.478 (0.318$0.701) \mathrm{mm}$ ] and well-formed, round or elliptical in shape, and almost invariably had a regular contour. Background ele-
\end{abstract}

\section{KARGER}

Fax +4161306 1234

E-Mail karger@karger.ch

www.karger.com (c) 2012 S. Karger AG, Basel

0025-7931/13/0853-0244\$38.00/0

Accessible online at:

www.karger.com/res ments lacked necrotic debris or exudate. The density [median (IQR) number of granulomas per slide: 6.85 (3.66-11) vs. $5.25(2.5-8), p=0.073$ ] and size [median (IQR) largest diameter: $0.51(0.319-0.733)$ vs. $0.398(0.318-0.522), p=0.071]$ tended to be larger in stage I than in stage II sarcoidosis. A necrotic background was common in the tuberculosis cohort studied (79 vs. $0 \%, p<0.0001$ ). Conclusions: Granulomas can be reliably identified on TBNA cytological material once their characteristic cytomorphology is delineated. A higher density of granulomas in lymphadenopathy of stage I sarcoidosis patients could partly explain the higher success rate constantly obtained by TBNA and EBUS-TBNA in this stage of the disease. A necrotic background suggests a tubercular etiology of the granulomas over a sarcoidal one, in the appropriate clinical setting.

Copyright $\odot 2012$ S. Karger AG, Basel

\section{Introduction}

Sarcoidosis is a multisystem disorder whose diagnosis is one of exclusion and is thought to be more accurate if the clinical and radiological suspicion is corroborated by the demonstration of nonnecrotizing granulomatous inflammation in at least one involved organ $[1,2]$. 
As many patients with sarcoidosis present only with identifiable intrathoracic changes, bronchoscopy has long been used to retrieve specimens for pathologic confirmation of clinically suspected disease. While transbronchial lung biopsy was the preferred method up to the mid-90s [3, 4], a growing body of literature data published in the past 15 years suggests that transbronchial needle aspiration (TBNA) of hilar and/or mediastinal lymph nodes, both conventional and ultrasound-guided, represents the bronchoscopic procedure associated with the highest yield in patients with stage I-II disease [5-9].

Early studies aimed at assessing the value of conventional TBNA in the diagnosis of sarcoidosis employed 19-gauge needle cores of tissue and required tissue with granulomatous inflammation [6, 10-12]. More recently, there has been a shift towards the use of TBNA cytology samples obtained using smaller gauge needles (21- and 22 -gauge). Despite several published studies relying on cytologic material obtained with TBNA and EBUSTBNA to diagnose both sarcoidal and tubercular lymphadenopathy $[8,9,13-22]$, clinical experience and literature data suggest that the interobserver variability in the identification of granulomas on cytological samples from intrathoracic lymphadenopathy can be considerable among pathologists $[23,24]$.

The aim of the present study was to describe the morphologic characteristics of sarcoidal granulomas on cytological samples retrieved with TBNA from intrathoracic lymphadenopathy and correlate these with the radiographic stage of the disease.

\section{Material and Methods}

\section{Study Design}

The study protocol was approved by the Ethics Committee of the Azienda USL di Bologna (protocol No. 802/CE). The files of the Pathology and Interventional Pulmonology Units of Maggiore Hospital (Bologna, Italy) were searched for all diagnoses of granulomatous inflammation made on TBNA specimens from hilar and mediastinal lymph nodes from January 1, 2005, and December 31, 2010. Two clinicians (M.P. and R.T.) blinded to the pathologic details of the TBNA specimens reviewed the medical and radiological charts of screened patients to verify whether the final diagnosis of sarcoidosis or tuberculosis was reliable, and to determine the stage of the disease (I or II) for the sarcoidosis patients. Besides the cytological findings, the diagnosis of sarcoidosis required a compatible clinical and radiological picture, whereas a definite diagnosis of tuberculosis required the presence of either a positive culture or a positive direct stain for acid-fast bacilli on a specimen from the airways or the intrathoracic nodes. A minimum follow-up length of 6 months was required for study entry. Selected patients with sarcoidosis or tuberculosis were then included in the study population and the control population, respectively, provided they had received neither steroids nor antimycobacterial treatment before bronchoscopy. At this point, two pathologists experienced in lung cytology (A.C. and K.O.L.), both blinded to the clinical-radiological details, reviewed all of the cytological hilar and mediastinal TBNA specimens and registered, for each patient, the following parameters regarding the granuloma: number per slide, largest and shortest diameter, shape, presence of giant cells, presence of fibrosis, and characteristics of the background. Possible disagreements between the two pathologists were resolved by consensus.

\section{Bronchoscopy Procedure and Handling of the Specimens}

TBNA was performed in the conventional, 'blind' fashion, with either 19-gauge or 22-gauge needles (MW-319 or MW-122; BARD Inc., Billerica, Mass., USA), during standard flexible bronchoscopy. Only lymph nodes with a short axis greater than $1 \mathrm{~cm}$ at CT were selected for aspiration. Each TBNA specimen was collected on clean glass slides. If a histologic core of tissue was obtained, it was gently removed from the slide and placed in a formalin solution. The remaining cytologic material was smeared on clean glass slides ('smear technique') which were half air dried and half ethanol fixed.

\section{Pathologic Evaluation}

Slides were stained with Papanicolaou, May-Grünwald-Giemsa, and Ziehl-Nielsen stains. Only adequate slides (i.e. slides in which $\geq 50 \%$ of the cellularity was constituted by nonneoplastic lymphocytes) were evaluated for the study. The mean number of granulomas per slide in each case was calculated by dividing the total number of granulomas by the number of adequate slides. The shape of granulomas, whether round (with comparable diameters) or elliptic (with one axis exceeding the other), was recorded. The minimum and maximum diameter of the granulomas was evaluated at a high magnification using a $\times 20$ or $\times 40$ objective with a $\times 10$ ocular lens and registered in millimeters. Furthermore, the characteristics of the background (clean, necrotic, or bloody) were noted; a necrotic background was characterized by the presence of nuclear debris, a bloody background was identified by the presence of abundant blood admist the group of cells and the tissue fragments, and a clean background was attributed to slides devoid of any of the previous features. The presence of other features, namely giant cells and fibrosis, was recorded. Only giant cells with an unequivocal cytologic appearance were accepted. Fibrosis was scored as 0,1 , or 2 , with 0 corresponding to no fibrosis, 1 to the presence of rare fragments of fibrous tissue, and 2 to several and/or large and coalescing fragments of fibrous tissue being observed.

\section{Statistical Analysis}

The Shapiro-Wilk test was used to test the normal distribution of quantitative variables; as they were not normally distributed, the results were expressed as medians and IQR (25th to 75th percentile) and comparisons between groups were done using the Mann-Whitney U test. Qualitative variables were summarized as numbers and percentages. The comparison among groups of qualitative variables was performed using a $\chi^{2}$ test or Fisher's exact test, as appropriate. Data analysis was performed using the Stata statistical package (release 11.1; Stata Corporation, College Station, Tex., USA). 
Table 1. Morphologic characteristics of sarcoidal and tubercular granulomas on cytologic TBNA specimens from intrathoracic nodes

\begin{tabular}{|c|c|c|c|}
\hline Median (IQR) number per slide & $6.4(3.5-10.3)$ & $4(2.08-6)$ & $0.049^{\S}$ \\
\hline Median (IQR) largest diameter, mm & $0.478(0.318-0.701)$ & $0.567(0.509-0.701)$ & 0.093 \\
\hline Median (IQR) shortest diameter, mm & $0.115(0.095-0.14)$ & $0.132(0.108-0.159)$ & 0.239 \\
\hline Shape, n (\%) & & & 0.863 \\
\hline Round & $62(50)$ & 6 & \\
\hline Presence of giant cells, n (\%) & $72(59)$ & $13(93)$ & $0.017^{\S}$ \\
\hline Presence of fibrosis, n (\%) & & & 0.254 \\
\hline No & $32(26)$ & $6(43)$ & \\
\hline Minimal fibrosis & $62(50)$ & $7(50)$ & \\
\hline Extensive fibrosis & $29(24)$ & $1(7)$ & \\
\hline Background, n (\%) & & & $<0.0001^{\S}$ \\
\hline
\end{tabular}

\section{Results}

The study population includes 123 sarcoidosis patients with a median (IQR) age of $43(36-54)$ years, a male-tofemale ratio of 1.1, and a I-to-II sarcoidosis stage ratio of 2.61. The control population was made up of 14 tuberculosis patients with a median (IQR) age of $34(24-41)$ years and a male-to-female ratio of 1.33 .

The prevalence of malignancy in the sarcoidosis population was as low as $3.25 \%(4 / 123)$. Three patients were being treated for malignancy (1 colonic cancer, 1 renal cancer, 1 breast cancer) when the diagnosis of sarcoidosis was established by bronchoscopy, whereas the fourth patient had been treated for ovarian cancer 2 years before bronchoscopy. The overall mean follow-up length for these 4 patients was 53 months after the diagnosis of sarcoidosis was established.

The mean number of adequate slides was 7.46 in sarcoidosis and 8.71 in tuberculosis, whereas the mean number of adequate slides showing granulomas was 4.92 in sarcoidosis and 5.28 in tuberculosis.

Table 1 summarizes the data on the main morphologic characteristics of sarcoidal and tubercular granulomas on cytological specimens obtained with TBNA from hilar and mediastinal lymphadenopathy.

Granulomas in cytological specimens from sarcoidosis patients are quite small [median (IQR) largest diameter: $0.478(0.318-0.701) \mathrm{mm}]$ and appear as well-defined, tiny nodules somehow 'popping out' of the slide, and they are easily appreciated even at low power (fig. 1a). They consist of a tight aggregate of histiocytes, round or elliptical in shape, with a regular contour (fig. 1b). The histiocytes are characteristically epithelioid, with twisted nuclei (fig. 1b). Their background is clean (fig. 1a), with only a minority of cases showing a hemorrhagic background. In no cases of the present series did the smears exhibit a thick and diffuse, hypocellular, necrotic background. Giant cells were present in approximately two thirds of the patients (fig. 1c) and fibrotic changes were observed in $74 \%$ of cases (fig. 1d).

Tubercular granulomas are quite large [median (IQR) largest diameter: $0.567(0.509-0.701) \mathrm{mm}]$ and usually have an irregular, uneven contour (fig. $2 \mathrm{a}, \mathrm{b}$ ). Compared to sarcoidosis, they have a significantly lower density [median (IQR) number per slide: 4 (2.08-6) vs. 6.4 (3.5$10.3) ; \mathrm{p}=0.049$ ] and are significantly more likely to show a necrotic background (79 vs. $0 \%, p<0.0001$ ), acid-fast bacilli ( 43 vs. $0 \%, \mathrm{p}<0.0001$ ), and giant cells ( 93 vs. $59 \%$, $\mathrm{p}=0.017)$ (fig. 2a, b).

Table 2 analyzes the possible correlations between the morphological characteristics of granulomas in sarcoidosis patients and the radiographic stage of the disease (I vs. II). Compared to stage II sarcoidosis, granulomas of stage I patients tend to have a higher density [median (IQR) number of granulomas per slide: 6.85 (3.66-11) vs. $5.25(2.5-8) ; \mathrm{p}=0.073$ ], tend to be bigger [median (IQR) largest diameter: $0.51(0.319-0.733)$ vs. $0.398(0.318-$ 

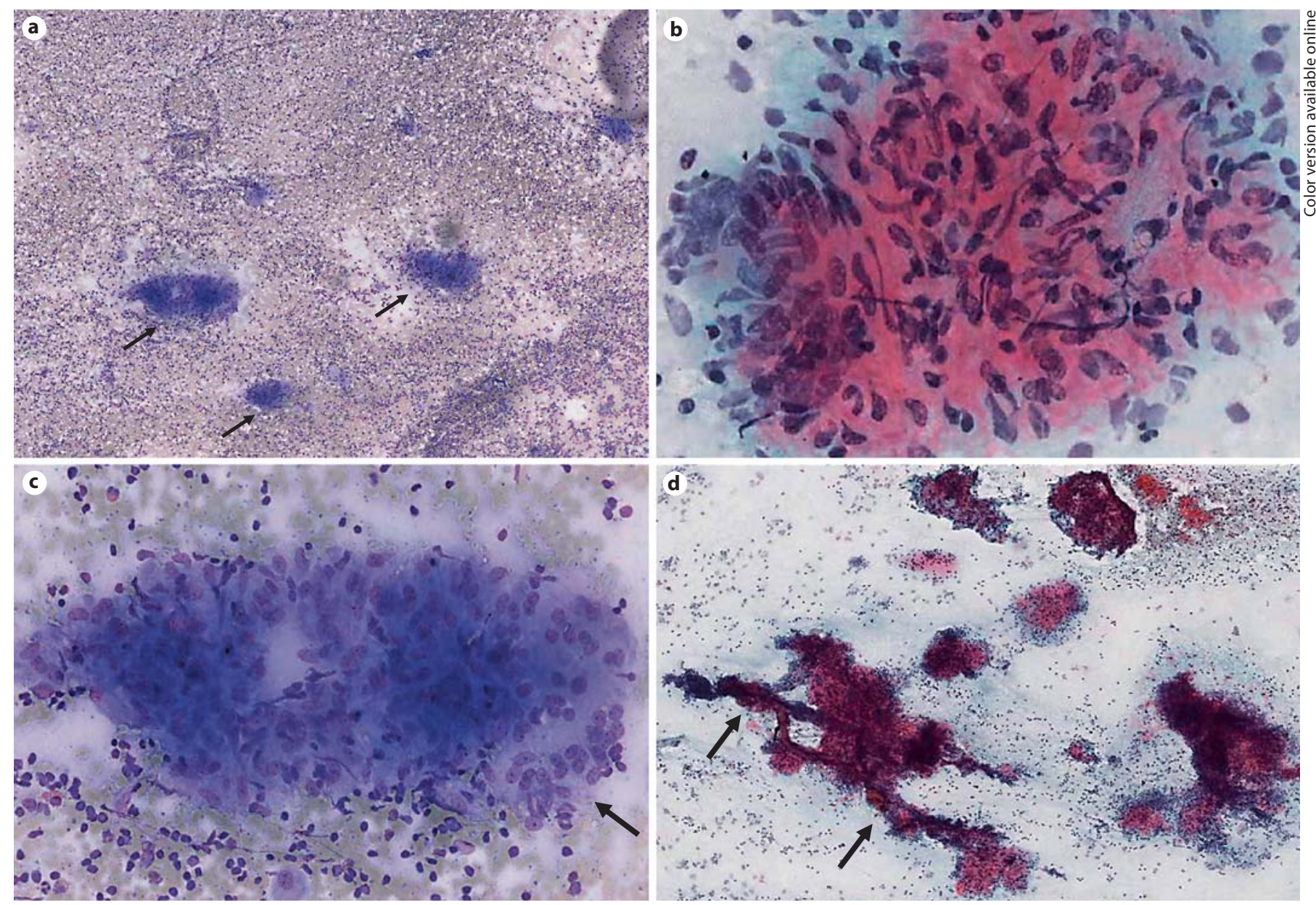

Fig. 1. TBNA cytological smears obtained from mediastinal sarcoidal lymph nodes. a Granulomas appear as well-defined nodules, 'popping out' of the slide (arrows). The background shows numerous lymphocytes and absence of necrotic debris or exudate. May-Grünwald-Giemsa. ×40. b A granuloma at a higher magnification shows a regular contour, with a tight aggregation of epi-

$0.522) ; \mathrm{p}=0.071]$, and are significantly more likely to have giant cells ( 59 vs. $38 \%$; $\mathrm{p}=0.005$ ).

We did not find any significant correlation between any of the morphologic parameters of the granuloma described above and the needle size used (table 3) or the patients' age (data not shown).

\section{Discussion}

The main finding of the present study is that the morphologic characteristics of granulomas allow them to be easily and reliably identified on the cytological material obtained with TBNA. Sarcoidal granulomas (fig. 1a-d)

thelioid histiocytes, with many having twisted nuclei. Papanicolaou. $\times 400$. c A multinucleated giant cell (arrow) is visible at the edge of an elliptical granuloma. May-Grünwald-Giemsa. $\times 200$. d Strands of fibrosis (arrows), along with several granulomas. Papanicolaou. $\times 40$.

appear as tiny 'nodules' consisting of a tight aggregate of epithelioid histiocytes. These nodules, which are easily appreciated even at low power, are well defined, round or elliptical in shape, and have a regular contour in most cases. Their background is usually clean, with only a minority of cases showing a hemorrhagic background. A necrotic, 'dirty' background was the only morphologic finding which proved specific enough to suggest a tubercular etiology of the granulomatous inflammation over a sarcoidal one in the present series, as already observed by Fritscher-Ravens et al. [14, 25] in two studies aimed at assessing the role of EUS-NA in sarcoidosis and tuberculosis. It should be kept in mind, however, that the presence of a necrotic background is useful only when present, as

Respiration 2013;85:244-251 
Table 2. Morphologic characteristics of granulomas on cytologic TBNA specimens from intrathoracic nodes according to the radiologic stage of sarcoidosis

\begin{tabular}{|c|c|c|c|}
\hline Characteristic of granulomas & $\begin{array}{l}\text { Sarcoidosis } \\
\text { stage I }(n=89)\end{array}$ & $\begin{array}{l}\text { Sarcoidosis } \\
\text { stage II }(n=34)\end{array}$ & $\mathrm{p}$ value \\
\hline Median (IQR) number per slide & $6.85(3.66-11)$ & $5.25(2.5-8)$ & 0.073 \\
\hline Median (IQR) largest diameter, mm & $0.51(0.319-0.733)$ & $0.398(0.318-0.522)$ & 0.071 \\
\hline Median (IQR) shortest diameter, mm & $0.115(0.095-0.134)$ & $0.127(0.101-0.14)$ & 0.239 \\
\hline \multicolumn{4}{|l|}{ Shape, n (\%) } \\
\hline Round & $44(49.5)$ & $18(53)$ & \multirow[t]{3}{*}{0.852} \\
\hline Elliptical & $12(13.5)$ & $5(15)$ & \\
\hline Both & $33(37)$ & $11(32)$ & \\
\hline Presence of giant cells, n (\%) & $59(59)$ & $13(38)$ & $0.005^{\S}$ \\
\hline \multicolumn{3}{|l|}{ Presence of fibrosis, $\mathrm{n}(\%)$} & \multirow[t]{4}{*}{0.14} \\
\hline No & $21(24)$ & $11(32)$ & \\
\hline Minimal fibrosis & $43(48)$ & $19(56)$ & \\
\hline Extensive fibrosis & $25(28)$ & $1(7)$ & \\
\hline \multicolumn{3}{|l|}{ Background, n (\%) } & \multirow[t]{4}{*}{0.466} \\
\hline Clean & $65(73)$ & $27(79)$ & \\
\hline Bloody & $24(27)$ & $7(21)$ & \\
\hline Necrotic & $0(0)$ & $0(0)$ & \\
\hline
\end{tabular}

$\S$ Statistically significant.

Table 3. Morphologic characteristics of granulomas on cytologic TBNA specimens from intrathoracic nodes according to needle type

\begin{tabular}{lccc}
\hline Characteristic of granulomas & 19-gauge $(\mathrm{n}=87)$ & 22-gauge $(\mathrm{n}=36)$ & p value \\
\hline Median (IQR) number per slide & $3.6(6.75-10)$ & $3.37(5.75-11)$ & 0.511 \\
Median (IQR) largest diameter, mm & $0.318(0.478-0.701)$ & $0.318(0.51-0.691)$ & 0.766 \\
Median (IQR) shortest diameter, mm & $0.83(0.115-0.14)$ & $0.96(0.127-0.153)$ & 0.124 \\
Shape, $\mathrm{n}(\%)$ & & & 0.354 \\
$\quad$ Round & $43(49.5)$ & $19(53)$ & $7(19)$ \\
$\quad$ Elliptical & $10(11.5)$ & $10(28)$ & 0.404 \\
$\quad$ Both & $34(39)$ & $19(53)$ & 0.074 \\
Presence of giant cells, $\mathrm{n}(\%)$ & $53(61)$ & $6(17)$ & \\
Presence of fibrosis, $\mathrm{n}(\%)$ & $26(30)$ & $17(47)$ & 0.379 \\
$\quad$ No & $45(52)$ & $13(36)$ & \\
$\quad$ Minimal fibrosis & $16(18)$ & $25(69.5)$ & \\
$\quad$ Extensive fibrosis & $67(77)$ & $11(30.5)$ & \\
Background, $\mathrm{n}$ (\%) & $20(23)$ & & \\
$\quad$ Clean & & & \\
$\quad$ Bloody & & & \\
\hline
\end{tabular}

the cytologic findings from tubercular lymphadenopathy may also include nonnecrotizing granulomas as well as necrotic, caseous material without granulomas [21, 22].

The simple aggregates of epithelioid histiocytes, which are extremely common in normal and reactive mediastinal lymph nodes, as well as the aggregates of bronchial cells, represent the main pitfalls for the diagnosis of gran- ulomas on cytology (fig. 3a, b). Compared to true granulomas, epithelioid histiocytes are loose and never form discrete nodules, whereas the aggregates of bronchial cells show cilia and/or terminal bars, often identifiable by focusing on their periphery.

Recognizing the morphologic features described above is of great importance because the diagnostic yield 

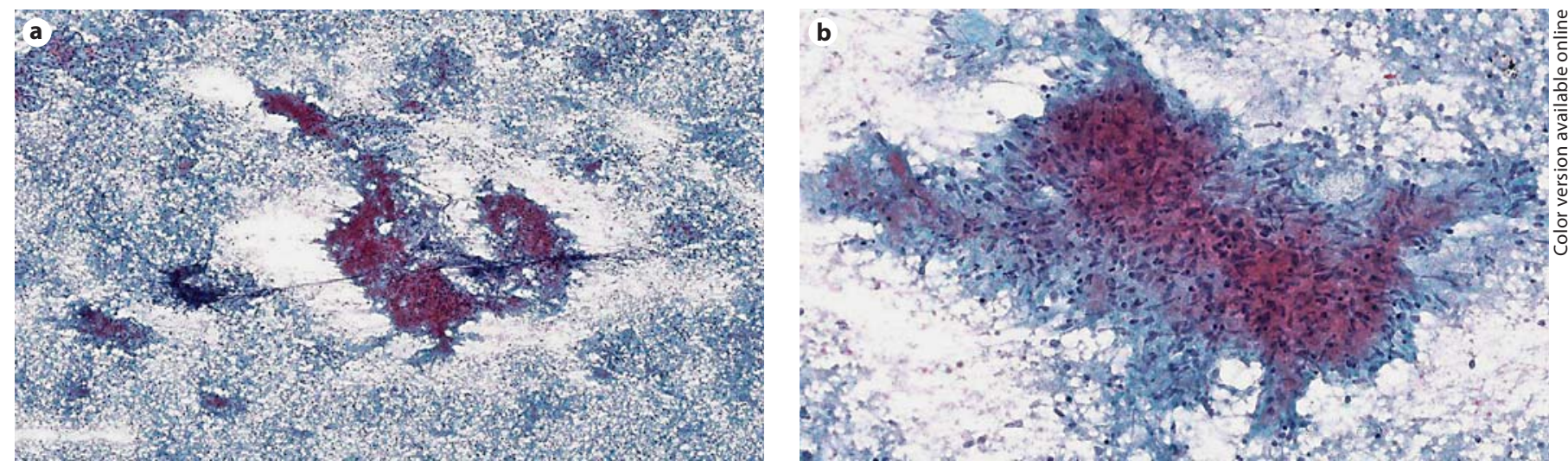

Fig. 2. TBNA cytological smears obtained from mediastinal tubercular lymph nodes. a A few granulomas superimposed on an extensively necrotic background. Papanicolaou. $\times 40$. b A large tubercular granuloma with an irregular contour. Papanicolaou. $\times 200$.
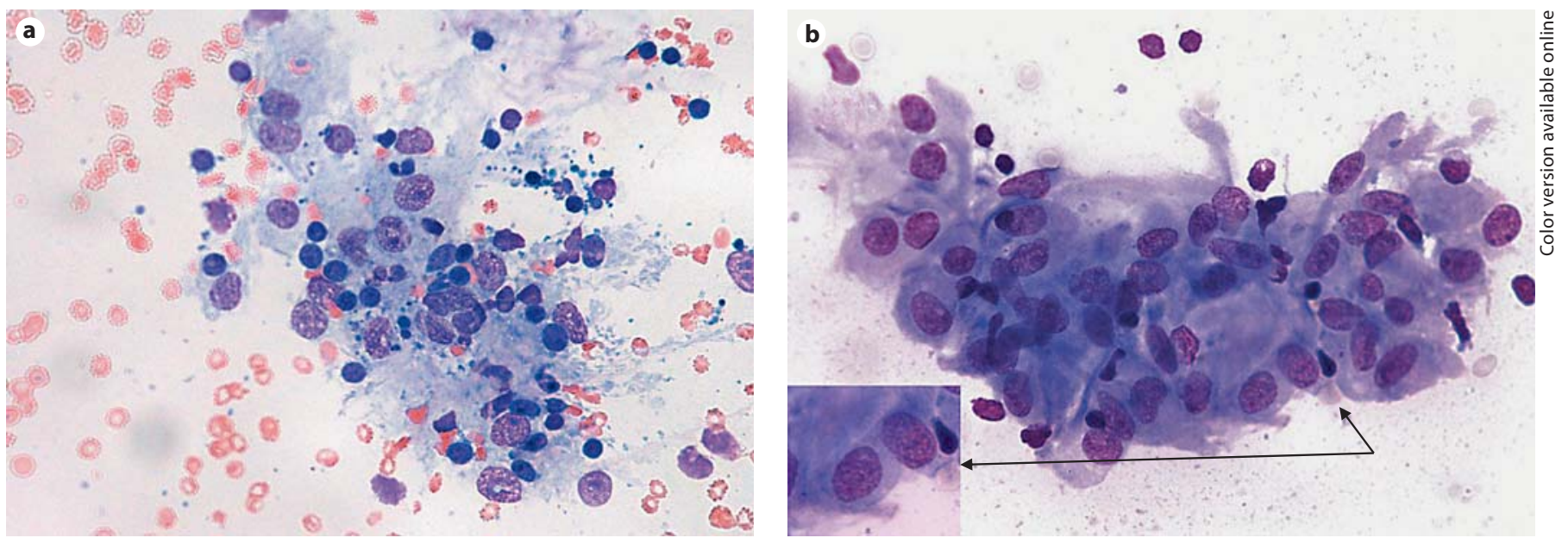

Fig. 3. Main pitfalls in the diagnosis of granulomas on TBNA cytological specimens. a A simple aggregate of epithelioid histiocytes. Compared to a true granuloma, such aggregates tend to be loose and never form discrete nodules. May-Grünwald-Giemsa. ×400. b A cohesive aggregate of bronchial columnar epithelial cells can be differentiated from a granuloma by the presence of cilia or, more frequently, terminal bars (arrow). In the inset, terminal bars are often identifiable at the periphery of the aggregate (arrow). May-Grünwald-Giemsa. $\times 400$; inset, $\times 600$.

of TBNA depends on the ability of pathologists to identify granulomas on cytological specimens. In a randomized controlled trial of conventional versus EBUS-guided TBNA in the diagnosis of sarcoidosis, for instance, Tremblay et al. [23] made the key observation that the identification of granulomas in the same cytological material was significantly different among the two pathologists involved in the study, both in the per-patient analysis and in the per-lymph node analysis [24]. In the more clinically important per-patient analysis, in particular, the yield of TBNA and EBUS-TBNA rose from 53 to $73 \%$ and from 83 to $95 \%$, respectively, when the cytologic material was examined by a pathologist experienced in lung diseases. Furthermore, clinical experience and literature data suggest that many interventional pulmonologists are not able to replicate in sarcoidosis the excellent results they obtain with TBNA in the setting of malignancy [20, 26]. One of the possible explanations for these findings is the fact that the ability to retrieve cytological samples from the mediastinal lymph nodes is quite a recent devel- 
opment, and the techniques that allow this sampling are either underused (conventional TBNA) or not yet widespread in every country because of costs (EBUS-TBNA and EUS-NA) [27]. As a consequence, the experience of many pathologists with the cytological material obtained from mediastinal lymphadenopathy may not be extensive, and this may be particularly true for sarcoidosis, whose prevalence in the vast majority of studies assessing the role of TBNA in the diagnosis of clinically unselected intrathoracic lymphadenopathy is constantly much lower than that of malignancy $[16,19,20,28,29]$. Specific training and experience with the cytological material obtained from sarcoidal lymphadenopathy with these diagnostic methods is therefore likely to be crucial for pathologists to improve their diagnostic yield and the reproducibility of their diagnoses, as already demonstrated in the setting of malignant lymphadenopathy [30].

Besides the pathologic interpretation of the samples, the preparation of the cytological material has been suggested by some authors to have some influence on the yield of conventional and ultrasound-guided TBNA in sarcoidosis. Schwartz et al. [31], for instance, recently reviewed the cytological material retrieved with EBUSTBNA from the mediastinum of 25 sarcoidosis patients. Interestingly, in no case were granulomas seen on the material directly smeared onto slides, on cytospins, and on ThinPrep ${ }^{\circledR}$ preparations, whereas the yield of the cellblock preparations was extremely high (24 of 25 cases, 96\%). In another retrospective study aimed at assessing the role of EUS-NA in sarcoidosis, von Bartheld et al. [32] processed the material with the smear technique in all 100 patients, but they also obtained a cell block in 76 of them. Interestingly, although the cell block showed granulomas in 6 of 18 patients (33\%) whose cytological smear evaluation was negative, cell block material was more commonly inadequate ( $29 \%$ of patients) than in conventional smears ( $8 \%$ of patients). In the absence of a study specifically designed to evaluate the impact of various preparation methods in this setting, the extent to which any observed differences are true rather than related to local preference and experience with a given method is difficult to establish. Furthermore, a review of the pertinent literature revealed that sarcoidal granulomas were reliably and effectively identified regardless of the fact that the material obtained with both conventional and ultrasound-guided TBNA was exclusively smeared onto slides [7-9, 13-21], partly smeared onto slides, and partly used to prepare cell blocks $[22,32,33]$, or processed as ThinPrep and cell blocks [22, 24].
In the last year we have very frequently used EBUSTBNA in patients with hilar and mediastinal lymphadenopathy. Interestingly, we have not seen any difference in the cytomorphology of granulomas in smears obtained with EBUS-TBNA as compared with those obtained with conventional TBNA in sarcoidosis patients, whereas we have not yet had the opportunity to perform EBUSTBNA in a patient with tuberculous lymphadenopathy.

In most of the previous studies that have looked at the success of the procedure according to the disease stage, the yield of TBNA, EBUS-TBNA, and EUS-FNA from intrathoracic adenopathy was shown to be consistently higher in stage I than in stage II sarcoidosis by a rate ranging from 7 to $36 \%[8,9,11,12,14,20,22,24]$. The observation in the present study that granulomas in the lymph nodes of stage I patients tended to be larger and to have a higher density than those in stage II may provide a plausible explanation for the extra yield of TBNA in stage I disease. We look forward to seeing if larger, prospective trials will reinforce or contradict this finding. It is of note, however, that the density of granulomas in lung tissue of sarcoidosis patients has been used to explain differences in the yield of transbronchial lung biopsy by race and radiographic stage. Burke et al. [34], in particular, found that the density of granulomas in transbronchial lung biopsy specimens was significantly higher in AfricanAmerican sarcoidosis patients than in whites, as well as in stage II and III compared to stage I [34].

The present study has limitations. First, a larger control population (tuberculosis patients) would certainly have had a positive impact on the statistical analysis. Unfortunately, the incidence of tuberculosis in Italy is low (7.66/100,000/year in 2008), and the vast majority of tuberculosis patients present with parenchymal disease. As a consequence, these patients are usually diagnosed with tuberculosis by sputum culture or washing procedures (bronchial washing, bronchoalveolar lavage) and are almost never sent to referral centers for mediastinal biopsy even when they have concomitant thoracic lymph node involvement. The single center nature of this trial further limited the possibility to recruit more tuberculosis patients. Second, we cannot rule out with certainty that a few cases of sarcoid-like reactions to malignancy were included in the sarcoidosis group, in spite of the very low prevalence of malignancy in this group (3.25\%).

In conclusion, granulomas from intrathoracic adenopathy can be easily and reliably identified in cytological specimens. A higher density of granulomas could be the reason for the higher yield constantly obtained by TBNA and EBUS-TBNA in stage I sarcoidosis, as com-
Cancellieri/Leslie/Tinelli/Patelli/ Trisolini 
pared with stage II. The presence of a necrotic background with compatible symptoms is the only morphologic finding that tends to rule out sarcoidosis and favors, in the appropriate clinical setting, a diagnosis of tuberculosis.

\section{Financial Disclosure and Conflicts of Interest}

None of the authors has conflicts of interest to disclose.

\section{References}

-1 American Thoracic Society: Statement on sarcoidosis. Am J Respir Crit Care Med 1999; 160:736-755.

2 Judson MA: The diagnosis of sarcoidosis. Clin Chest Med 2008;29:415-427.

3 Gilman MJ, Wang KP: Transbronchial lung biopsy in sarcoidosis: an approach to determine the optimal number of biopsies. Am Rev Respir Dis 1980;122:721-724.

4 Poe RH, Israel RH, Utel MJ, et al: Probability of a positive transbronchial lung biopsy result in sarcoidosis. Arch Intern Med 1979; 139:761-763.

5 Bilaceroglu S, Mehta AC, Light R: Transbronchial needle aspiration for diagnosis of sarcoidosis. J Bronchol 2004;11:54-61.

-6 Morales MC, Patefield AJ, Strollo PJ, et al: Flexible transbronchial needle aspiration in the diagnosis of sarcoidosis. Chest 1994;106: 709-711.

-7 Trisolini R, Lazzari Agli L, Cancellieri A, Poletti V, Candoli P, Paioli D, et al: Transbronchial needle aspiration improves the diagnostic yield of bronchoscopy in sarcoidosis. Sarcoidosis Vasc Diffuse Lung Dis 2004;21: 147-151.

8 Trisolini R, Tinelli C, Cancellieri A, et al: Transbronchial needle aspiration in sarcoidosis: yield and predictors of a positive aspirate. J Thorac Cardovasc Surg 2008; 135 :837842.

-9 Nakajima T, Yasufuku K, Kurosu K, et al: The role of EBUS-TBNA for the diagnosis of sarcoidosis - comparison with other bronchoscopic diagnostic modalities. Resp Med 2009;103:1796-800.

10 Pauli G, Pelletier A, Bohner C, et al: Transbronchial needle aspiration in the diagnosis of sarcoidosis. Chest 1984;84:571-576.

11 Leonard C, Tormey VJ, O’Keane CO, Burke CM: Bronchoscopic diagnosis of sarcoidosis Eur Resp J 1997;10:2722-2724.

12 Bilaceroglu S, Perim K, Gunel O, Cagirici U, Buyuksirin M: Combining transbronchial aspiration with endobronchial and transbronchial biopsy in sarcoidosis. Monaldi Arch Chest Dis 1999;54:217-223.

13 Trisolini R, Lazzari Agli L, Cancellieri A, Poletti $\mathrm{V}$, Tinelli $\mathrm{C}$, Baruzzi $\mathrm{C}$, et al: The value of flexible transbronchial needle aspiration in the diagnosis of stage I sarcoidosis. Chest 2003; $124: 2126-2130$.
14 Fritscher-Ravens A, Sriram PVJ, Topalidis T, 25 Fritscher-Ravens A, Ghanbari A, Topalidis Hauber Hp, Meyer A, Soehendra N, et al: Diagnosing sarcoidosis using endosonography-guided fine needle aspiration. Chest 2000;118:928-935.

15 Annema JT, Vaselic M, Rabe KF: Endoscopic ultrasound-guided fine-needle aspiration for the diagnosis of sarcoidosis. Eur Resp J 2005:25:405-409.

16 Wildi SM, Judson MA, Fraig M, Fickling WE, Schmulewitz N, Varadarajulu S, et al: Is endosonorography guided fine needle aspiration (EUS-FNA) as good as we think? Tho$\operatorname{rax}$ 2004;59:794-799.

17 Wong M, Yasufuku K, Nakajima T, Herth FJ, Sekine J, Shibuya K, et al: Endobronchial ultrasound: new insight for the diagnosis of sarcoidosis. Eur Resp J 2007;29:1182-1186.

18 Garwood S, Judson MA, Silvestri G, Hoda R, Fraig M, Doelken P: Endobronchial ultrasound for the diagnosis of pulmonary sarcoidosis. Chest 2007;132:1298-1304.

19 Sharafkhaneh A, Baaklini W, Gorin AB, Green L: Yield of transbronchial needle aspiration in diagnosis of mediastinal lesions. Chest 2003;124:2131-2135.

20 Cetinkaya E, Yildiz P, Kadacal F, et al: Transbronchial needle aspiration in the diagnosis of intrathoracic lymphadenopathy. Respiration 2002;69:335-338.

21 Bilaceroglu S, Gunel O, Eris N, Cagirici U, Mehta AC: Transbronchial needle aspiration in diagnosing intrathoracic tuberculous lymphadenitis. Chest 2004;126:259-267.

-22 Navani N, Molyneaux PL, Breen RA, et al: Utility of endobronchial ultrasound-guided transbronchial needle aspiration in patients with tuberculous intrathoracic lymphadenopathy: a multicentre study. Thorax 2011; 66:889-893.

23 Tremblay A, Stather DR, Maceachern P, Khalil M, Field SK: A randomized controlled trial of standard versus endobronchial ultrasonography-guided transbronchial needle aspiration in patients with suspected sarcoidosis. Chest 2009;136:340-346.

24 Chee A, Khalil M, Stather D, MacEachern P, Field SK, Tremblay A: Cytologic assessment of endobronchial ultrasound-guided TBNA in sarcoidosis. J Bronchol Intervent Pulmonol 2012;19:24-28. T, et al: Granulomatous mediastinal adenopathy: can endoscopic ultrasound-guided fine-needle aspiration differentiate between tuberculosis and sarcoidosis? Endoscopy 2011;43:955-961.

26 Medford AR, Agrawal S, Free CM, Bennet JA: A prospective study of conventional transbronchial needle aspiration: performance and cost-utility. Respiration 2010;79: 482-489.

27 Haponik EF, Cappellari JO, Chin R, et al: Education and experience improve transbronchial needle aspiration performance. Am J Resp Crit Care Med 1995;151:1998-2002.

28 Trisolini R, Cancellieri A, Tinelli C, et al: Rapid on-site evaluation of transbronchial aspirates in the diagnosis of hilar and mediastinal adenopathy: a randomised trial. Chest 2011;139:395-401.

29 Herth FJ, Eberhardt R, Vilmann P, Krasnik M, Ernst A: Real-time endobronchial ultrasound guided transbronchial needle aspiration for sampling mediastinal lymph-nodes. Thorax 2006;61:795-798.

- 30 Skov BG, Baandrup U, Jakobsen GK, et al: Cytopathologic diagnoses of fine-needle aspirations from endoscopic ultrasound of the mediastinum: reproducibility of the diagnoses and representativeness of aspirates from lymph nodes. Cancer Cytopathol 2007;111: 234-241.

31 Schwartz LE, Griffin AC, Baloch Z: Cell block interpretation is useful in the diagnosis of granulomas on cytology. Diagn Cytopathol 2012;40:939-940

32 Von Bartheld MB, Veselic-Charvat M, Rabe KF, Annema JT: Endoscopic ultrasoundguided fine-needle aspiration for the diagnosis of sarcodosis. Endoscopy 2010;42:213217.

33 Tournoy KG, Bolly A, Aerts JG, et al: The value of endoscopic ultrasound after bronchoscopy to diagnose thoracic sarcoidosis. Eur Respir J 2010;35:1329-335.

34 Burke RR, Stone CH, Havstad S, Rybicki BA: Racial differences in sarcoidosis granuloma density. Lung 2009;187:1-7. 